\title{
Highlighting Strengths in Response to Discrimination: Developing and Testing an Allyship Positive Psychology Intervention
}

\author{
Meg A. Warren · Tejvir Sekhon · Rachael J. Waldrop
}

\begin{abstract}
Allyship is gaining popularity as a tool to combat discrimination. We developed and tested a novel allyship positive psychology intervention (PPI). Importantly, we examined observers' perceptions of intervention effectiveness given that observers represent the majority in many settings. Study $1(\mathrm{~N}=787)$ tested an intervention that highlighted a female employee's identity-related strengths following a discrimination episode. Compared to communicating an organization's diversity policy or confronting the transgressor, highlighting the target's identity-related strengths was rated higher in terms of inclusion and vitality engendered in the target. Mediation analyses indicated that highlighting strengths was perceived as boosting the target's vitality by signaling the ally's sincerity and prompting inclusion. In Study $2(\mathrm{~N}=802)$, amongst various types of identity-related strengths, highlighting the target's psychological and intellectual capital was as effective as highlighting all types of identity-related strengths combined, due to perceived sincerity. Thus, this research offers a quick, parsimonious, actionable, and non-confrontational allyship PPI.
\end{abstract}

Keywords: Allyship; strengths; intergroup processes; workplace; microaggressions; positive psychology intervention

\section{Introduction}

In the wake of heightened public consciousness (e.g., \#BlackLivesMatter movement, \#MeToo) surrounding racism and sexism, an increased sensitivity to the wellbeing of marginalized communities has led to calls for positive psychology to meaningfully address social, ecological, and systemic issues (Lambert et al., 2020; Warren \& Bordoloi, 2020; Waters et al., 2021). Although past calls for research on wellbeing of marginalized groups (e.g., Rao \& Donaldson, 2015) have resulted in increased attention to the strengths of marginalized groups (Jeglic et al., 2016; Vaughan et al., 2014), unintentional burdens have been placed on already overburdened groups (e.g., racial minorities in the U.S.) and community-based institutions (e.g., non-profits, schools) to build strengths, resilience and wellbeing in the face of prejudice and discrimination, inviting the frustration of critics (Yakushko \& Blodgett, 2021). An alternative, understudied approach is to understand how positive psychology can directly engage individuals with power (e.g., racial majorities in the U.S., corporate leaders) in combating prejudice and discrimination. In response to these calls and critiques, we propose a path forward by considering a positive psychological approach to equity and inclusiveness through allyship.

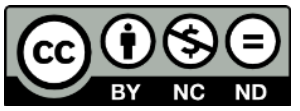

Copyright belongs to the author(s) www.internationaljournalofwellbeing.org 


\subsection{Allyship for equity and inclusiveness}

Broadly, allyship describes the actions of "a member of the 'dominant' or 'majority' group who works to end oppression in [their] personal and professional life through support of, and as an advocate for, the oppressed population" (Washington \& Evans, 1991, p. 195). Although an act of allyship is intended to support the victim, the potential impact of allyship is much broader. Allyship serves a relational function by creating inclusive micro-climates in institutions (Warren et al., 2019). Particularly, when enacted by a leader, allyship has the potential to both educate the perpetrator (Czopp \& Monteith, 2003) and model appropriate behaviors for observers, serving as a catalyst for change within the circle of the leader's influence. In this paper, we focus on one particular form of allyship - by men toward women. We frame male allyship as a tool to leverage male identity as a strength to support the wellbeing of women (Warren, Bordoloi, \& Warren, 2021).

Intervening as an ally in the face of discrimination is arguably uncomfortable, but interventions by bystanders yield fewer costs and more benefits as compared to interventions undertaken by the targets themselves (Czopp \& Monteith, 2003; Gulker et al., 2013). Furthermore, their position of authority grants leaders additional interpersonal power that can be used to facilitate a successful intervention. Thus, leader-allies may be in a unique position to intervene on behalf of marginalized group members while also experiencing lower interpersonal costs. Importantly, when a leader-ally intervenes, it serves to not only correct the perpetrator but also signal to observers, who often represent the majority in many settings, that microaggressions are not acceptable, thereby impacting institutional culture. As such, the purpose of this paper is to examine the effect of allyship interventions from the standpoint of observers.

\subsection{Existing allyship intervention: Communicating diversity and inclusion policy}

The development and testing of allyship interventions can equip dominant group individuals with concrete strategies to be effective in their roles as allies. Although there is growing interest in the potential contributions of allies in the workplace (Crary, 2017; Erskine \& Bilimoria, 2019), the impact and effectiveness of allyship actions and their underlying mechanisms are unclear. One likely reason is the assumption that the presence and enactment of an organization's human resources (HR) policies would serve to sufficiently reduce discrimination. In this way, the role of leaders is perceived as enacting, communicating and monitoring the implementation of such policies and practices (Den Hartog, Boon, Verburg, \& Croon, 2013; Purcell \& Hutchinson, 2007). After witnessing or being informed of prejudiced behavior, a leader may enact allyship by reminding the perpetrator about the company's HR policies on the matter. Indeed, within a phenomenological assessment of interviews, DeTurk (2011) found that allies commonly enacted policy in response to workplace discrimination as a rhetorical strategy to balance both their motivation to enact allyship and their concerns about preserving workplace decorum.

Despite the good intentions of such rhetorical strategies, leaders who simply instate organizational policy as allyship are embracing a relatively passive role (Buengeler et al., 2018). Given the intricate interpersonal and emotional context of workplace confrontations, this approach offers a limited repertoire of strategies for what leaders from socially dominant groups can do to actively support marginalized group employees. Furthermore, although enforcement of punitive policies may serve to subsequently deter explicit discriminatory behaviors, it is unlikely to inspire change among perpetrators, foster wellbeing for the targets, or cultivate an environment of cohesiveness in the organization-all of which are components of authentic allyship (Brooks \& Edwards, 2009; Sue, 2017). In fact, top-down enactment of diversity policies can provoke backlash from confronted perpetrators (Kidder et al., 2004), increase discomfort 
among dominant group employees in relating with co-workers from marginalized groups (Baba \& Hebert, 2004), and worsen attitudes toward marginalized groups (Hood et al., 2001). To better understand and develop potentially effective interventions, we next examine social psychological approaches to allyship interventions. In addition, we experimentally study the effectiveness of communicating policy versus other interventions discussed below.

\subsection{Existing allyship intervention: Confronting prejudiced behaviors}

Most social psychological research on micro-interventions by allies is framed as confrontation of prejudice and discrimination. Numerous studies show that confrontation of bias and prejudice is effective in reducing prejudice (see Mallet \& Monteith, 2019). For instance, confronted participants are less likely to express stereotypes and prejudiced attitudes (Ashburn-Nardo et al., 2008; Czopp et al., 2006). Calm confrontations that directly implicate the perpetrator can reduce backlash against the ally, maximize culpability of the perpetrator, and elicit future confrontation behaviors by observers (Martinez et al., 2017). Finally, the effects of confrontations last for some time, as seen in one study where confronted participants used fewer stereotypes and inhibited prejudiced behavior one week later (Chaney \& Sanchez, 2018).

Despite its effectiveness in holding perpetrators accountable, confrontation comes at interpersonal costs - it elicits negative emotions toward and negative evaluations of the confronter (Czopp et al., 2006). Failing to confront, however, also carries intrapersonal costs. In a series of studies, motivated bystanders who failed to confront prejudice reported more favorable attitudes towards the perpetrator and less support for confronting down the road, fueling selfpreservation rather than allyship (Rasinski et al., 2013). Without proper preparation for the potential interpersonal and intrapersonal risks that may accompany confrontation, sustained action and advocacy are likely to suffer (Sue, 2017). Given that confrontation may be a useful but bitter pill, we consider the effectiveness of confrontation as one allyship strategy in comparison to other allyship strategies but are primarily interested in developing and comparing the effectiveness of an additional positive psychology-based intervention that may incur fewer negative consequences for allies.

\subsection{New allyship positive psychology intervention: Highlighting minority resources}

Positive psychology interventions (PPIs) are tools that cultivate positive subjective experiences, support the development of positive traits, or create positive institutions (Meyers et al., 2013). To develop an intervention in response to microaggressions that will maximize positive subjective experiences and the construction of positive institutional culture, we consider an allyship microintervention grounded in a positive psychological framework.

Cha and Roberts (2019) propose that individuals from minority or underrepresented groups (in terms of sex, race, social class, etc.) utilize identity-based strengths (e.g., unique cultural knowledge) as resources to achieve desirable organizational outcomes. However, their identity mobilization framework notes that the potential for leveraging minority identities as resources can be disrupted by identity-related tensions. For example, a racial minority journalist can leverage their identity-related insights (i.e., knowledge and perspectives based on their experiences as a minority group member) to provide a different perspective on a presidential election, but may face challenges due to certain tensions such as pigeonholing (fear of being perceived as capable of only succeeding at minority-related work), activism tension (fear of being perceived as disruptive), objectivity tension (fear of being perceived as biased towards one's minority group) or perpetuation tension (fear of reinforcing existing stereotypes about one's 
group; Cha \& Roberts, 2019). Thus, reframing minority identities as a resource has potential yet leveraging them effectively can be a complex and tension-inducing process.

In the present work, we build on Cha and Roberts' (2019) influential work and circumvent tensions placed on minority individuals during identity mobilization by examining the effects of a leader-ally highlighting minority individuals' identity-based strengths as resources following a discrimination episode. From a social lens, this intervention shifts the burden away from minority individuals and focuses on leaders' opportunities to highlight resources and strengthen perceptions of minority individuals within an organization. To be sure, under some circumstances, highlighting a minority individual's identity may be experienced as coercive by the minority individual, who may not have consented to such visibility (Creary et al., 2015). However, highlighting these resources as an intervention directly following a microaggression that has already negatively highlighted a target's minority identity avoids these coercive implications. In fact, because the microaggression has already framed the minority identity as a liability, the leader remedies the situation by negating this and reframing the minority identity as a potential resource. As such it also serves to signal to minority groups that they may bring their minority identity-relevant authentic selves to work, if they so wish (Cha et al., 2019).

A recent qualitative study explored women's and men's perspectives of different strategies used by exceptional allies in the workplace (Warren \& Bordoloi, 2021). Promoting the impression of women by highlighting their strengths in front of colleagues emerged as one of the most memorable strategies used by allies, according to both men and women. Given the favorability between both potential allies and targets, the current paper extends this work by testing whether this strategy functions as an effective positive allyship intervention, compared to other contemporary interventions like communicating policy or confrontation.

\subsection{Boosting inclusion and vitality}

Within the existing literature, the end goal of allyship interventions is primarily to reduce negative characteristics of the perpetrator (e.g., prejudiced attitudes) and ultimately reduce negative subjective experiences for marginalized group members (e.g., feelings of exclusion). By applying a positive psychology perspective, we investigate from observers' perspectives how allyship interventions might improve positive subjective experiences and overall wellbeing for marginalized group members. Specifically, we observe how two concepts, inclusion and vitality, function within this intervention paradigm.

In the diversity literature, researchers conceptualize inclusion as the degree to which marginalized group members feel a sense of belonging, uniqueness, and comfort when expressing their authentic selves (Shore et al., 2011; Jansen et al., 2014). Feeling included is thus an important aspect of workplace wellbeing, particularly within diverse organizations. Microaggressions threaten group cohesiveness by compromising feelings of inclusion; therefore, to be effective, an intervention must serve to restore or increase the subjective experience of felt inclusion. In the present studies, we examine whether observers believe that an ally's intervention results in feelings of inclusion for the target.

Vitality is a feeling of liveliness and energy and is an aspect of psychological wellbeing that is associated with key desirable organizational outcomes such as work engagement and creativity (Bakker et al., 2020; Ryan \& Frederick, 1997). In contrast, discrimination predicts adverse consequences for marginalized group members such as depression, anxiety, stress, psychological distress, self-esteem, and poorer life satisfaction (see meta-analyses, Pascoe \& Richman, 2009; Triana et al., 2015), and over time, predicts disengagement from the workplace (Foster, 2009; Major \& Schmader, 1998). Beyond reducing the psychological and occupational distress 
associated with workplace discrimination, we believe that an effective allyship intervention should reinstate a feeling of vitality. Therefore, in the current studies, we compare and test the effectiveness of allyship interventions in predicting marginalized group members' sense of vitality.

Across two studies, we tested observers' perceptions of the relative effectiveness of three theoretically-derived allyship interventions in boosting women's feelings of inclusion and vitality. First, we contrasted our novel intervention of highlighting minority identity resources against the existing recommended interventions. Next, we tested the relative effectiveness of highlighting different types of minority identity resources.

\section{Study 1: Impact of resource highlighting}

In Study 1, we compared the impact of resource highlighting, confronting, and communicating corporate policy as a response to discrimination against a pregnant female employee by measuring observers' feelings of inclusion and vitality. We predicted that an allyship intervention would primarily foster feelings of inclusion, which in turn would lead to feelings of vitality.

\subsection{Method}

Eight hundred and three working adults from a US-based online panel participated for payment. The project received IRB exemption and participants provided informed consent. Sensitivity analyses indicate that this sample size is sufficient to detect a small-to-medium effect size at $80 \%$ power (G*Power; Faul et al., 2007). After excluding data from six participants who either chose 'other' for gender or did not answer the question, the final sample size for this study was 797 participants (391 female, 406 male; Median age $=35$ ). All participants read the following scenario about a meeting:

"John, the VP of Sales at FitWell Shoes, was holding his Monday morning briefings. All four regional managers (Susan, David, Mike, and Bob) who report to John were seated at the conference table and took turns sharing highlights of their weekend before starting the meeting. When it was Susan's turn, she exclaimed, "My big news is that we just found out this weekend that I'm pregnant!"

Participants were randomly assigned to one of six between-subjects conditions (no transgression, no intervention, stating policy, confrontation, resource highlighting, and hybrid [confrontation + resource highlighting]). The only difference between the six conditions was the comment that a male colleague made and the leader's response. In the "no transgression" condition, there was no sexist comment. In the "no intervention" condition, a male colleague made a sexist comment about the target's (i.e., Susan's) pregnancy but the leader did not react. In the "stating policy" condition, the leader responded to the sexist comment by stating the company's official policy that they do not discriminate based on motherhood status. In the "confrontation" condition, the leader pointed out that the male colleague's comment was sexist. In the "resource highlighting" condition, the leader responded to the sexist comment by highlighting the resources that the target would bring to the organization due to her motherhood experiences. In the "hybrid (confrontation + resource highlighting)" condition, the leader highlighted the target's resources while calling out the male colleague's comment as sexist. The stimuli for these six conditions are detailed in the appendices.

After reading the respective scenario, participants indicated their inferences (on a 9-point scale) regarding Susan's feelings of vitality and inclusion in her relationship with the leader (John). Vitality was measured by adapting four relevant items from the Vitality subscale of the 
High-Quality Relationships at Work (HQR) scale (Carmeli, 2009). The items: "I feel enthusiastic about working with him", "when I am working with him, I feel positive energy", "working with him makes me feel good", and "I look forward to my interactions with him." ( $\alpha=0.98)$ were presented along with the question "To what extent do you think that Susan would agree with the following statements about John?"1 Inclusion was measured by adapting four relevant items of the Perceived Group Inclusion Scale (PGIS; Jansen et al., 2014), which measures relationshipspecific inclusion with male colleagues. The items, "He treats me like an insider", "he appreciates me", "he allows me to be authentic", and "he encourages me to present myself the way I am" ( $\alpha$ $=0.97$ ) were presented along with the question "To what extent do you think that Susan would agree with the following statements about John?" Of note, a wealth of research shows that the quality of employees' relationship with their leaders/supervisors is an important predictor of employees' job satisfaction (see review, Fisk \& Friesen, 2012) and wellbeing (e.g., Ellis et al., 2019). Thus, our contextualized measure that captures vitality and inclusion experienced by Susan in her relationship with John offers greater nuance over extant measures of vitality and inclusion. ${ }^{2}$ Finally, participants answered demographic questions.

\subsection{Results}

\subsubsection{Perceived inclusion}

A factorial analysis of variance (ANOVA) found a main effect of condition $(F(5,785)=64.37, p<$ $\left..01, \eta_{p}{ }^{2}=.29\right)$ and a marginal main effect of gender $\left(F(1,785)=2.84, p=0.092, \eta_{p^{2}}=.004\right)$. Planned contrasts revealed that, among the four interventions, perceived inclusion was higher in the highlighting resources condition $(M=7.42, S D=1.80)$ than both the stating policy $(M=6.5, S D=$ 2.13) and confrontation condition $(M=6.82, S D=2.06)$ (both $p s<0.05)$ but not different than the hybrid (confrontation + highlighting resources) condition $(M=7.24, S D=2.03)$. Perceived inclusion was lowest in the no intervention condition $(M=3.79, S D=2.32)$ compared to all other conditions (all $p \mathrm{~s}<0.001$ ). Perceived inclusion was also lower when there was no transgression $(M=5.32, S D=1.97)$ compared to any type of intervention post transgression (all $p s<0.001)$. Overall, women perceived marginally higher inclusion $(M=6.31, S D=0.10)$ than men $(M=6.02$, $S D=0.10)$.

Interestingly, there was a statistically significant interaction between gender and condition $\left(F(5,785)=4.45, p<0.01, \eta p^{2}=.028\right)$ on perceived inclusion. See Table 1 (below) for descriptive statistics and Figure 1 for interaction effects. Women $(M=3.30, S E=2.17)$ perceived less inclusion than men $(M=4.23, S E=2.37)$ in the no intervention condition $(F(1,785)=7.18, p<0.01)$, but more inclusion $(M=7.97, S E=1.64)$ than men $(M=6.65, S E=2.19)$ in the hybrid condition $(F(1,785)=$ $11.80, p<0.01)$. Though we did not predict an interaction, it is not surprising that women perceived Susan to have lower feelings of inclusion when there was no intervention but perceived

\footnotetext{
${ }^{1}$ We chose to include a specific measure of vitality rather than a more well-established general measure of relationship quality (Leader-Member Exchange; LMX) or satisfaction with one's supervisor because these are conceptually distinct. LMX measures respect, trust, and obligation towards one's supervisor/leader which may not be particularly energizing and could insinuate that allyship creates obligation. Vitality reflects a positive, high arousal emotional state that is experienced as energizing (Kark \& Carmeli, 2009). We anticipate that feelings of vitality (but not LMX) are an important antidote to the fatigue and depletion associated with discrimination. Feelings of vitality are also distinct from satisfaction with one's supervisor. Simply, one may feel satisfied with a supervisor's actions if one may not have high expectations from them, but one may also not feel energized because of those actions. This difference may be particularly meaningful in the distress-producing context of discrimination.

2 The relevance and use of similar adaptations to measure vitality and inclusion as outcomes of an allyship relationship have been demonstrated across multiple studies (e.g., Warren, Bordoloi, \& Warren, 2021; Warren \& Schwam, 2021; Warren, Sekhon et al., 2021).
} 
her to have higher feelings of inclusion in the hybrid condition, indicating potentially higher levels of empathy based on their own experiences as compared to men.

\section{Figure 1. Perceived inclusion in the relationship with the leader in Study 1}

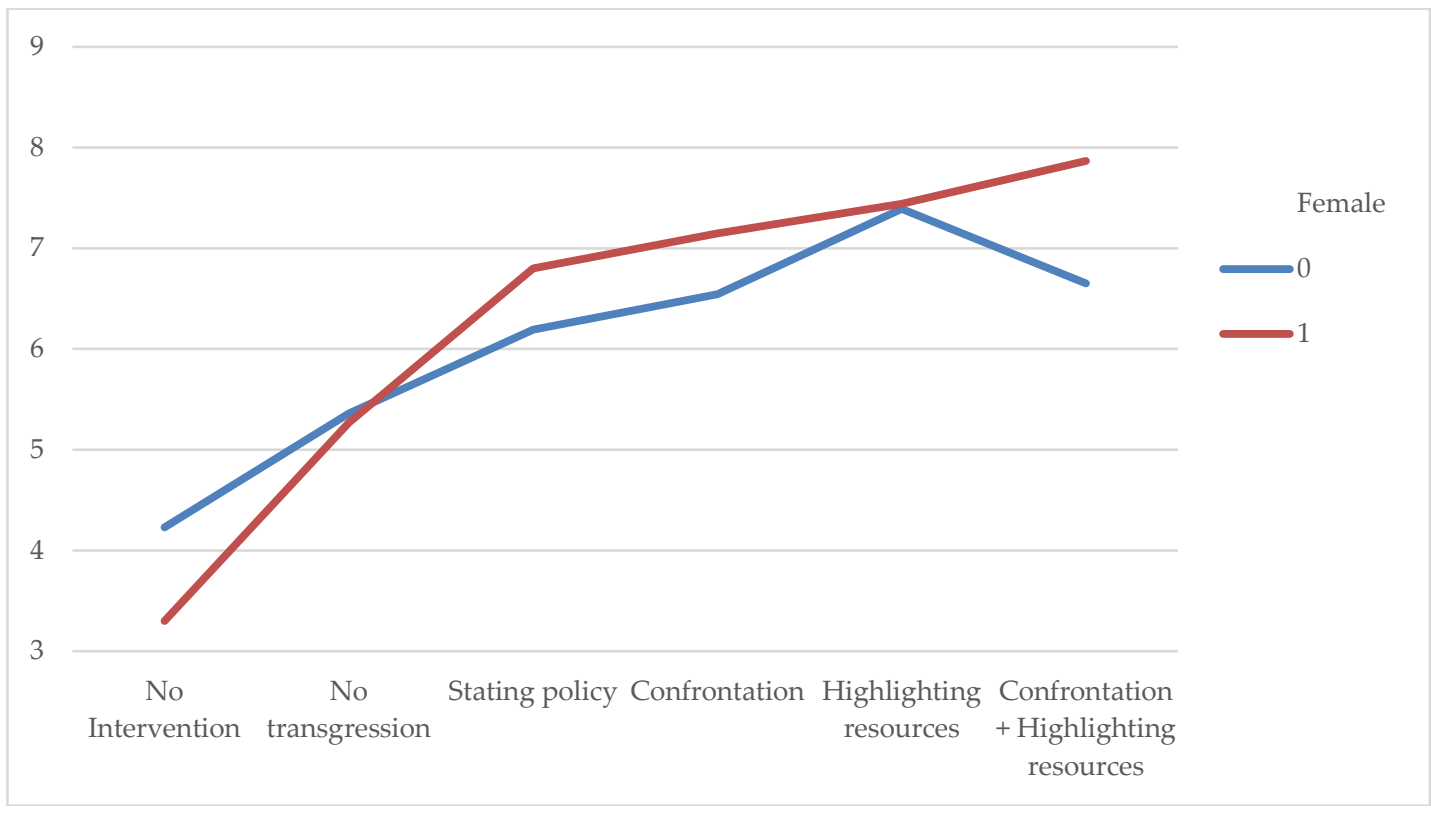

Table 1. Descriptive statistics for Study 1

\begin{tabular}{|c|c|c|c|c|}
\hline \multirow[t]{2}{*}{ Conditions } & \multicolumn{2}{|c|}{$\begin{array}{c}\text { Perceived } \\
\text { inclusion }\end{array}$} & \multicolumn{2}{|c|}{$\begin{array}{l}\text { Perceived } \\
\text { vitality }\end{array}$} \\
\hline & Male & Female & Male & Female \\
\hline \multirow[t]{2}{*}{ No intervention } & 4.23 & 3.30 & 4.17 & 3.28 \\
\hline & 2.37 & 2.17 & 2.35 & 2.18 \\
\hline \multirow[t]{2}{*}{ No transgression } & 5.36 & 5.27 & 5.19 & 5.09 \\
\hline & 2.18 & 1.74 & 2.27 & 1.78 \\
\hline \multirow[t]{2}{*}{ Stating policy } & 6.19 & 6.80 & 6.18 & 6.77 \\
\hline & 1.96 & 2.27 & 2.03 & 2.25 \\
\hline \multirow[t]{2}{*}{ Confrontation } & 6.54 & 7.15 & 6.61 & 7.17 \\
\hline & 2.09 & 1.98 & 2.15 & 1.97 \\
\hline \multirow[t]{2}{*}{ Highlighting resources } & 7.39 & 7.44 & 7.50 & 7.54 \\
\hline & 1.80 & 1.81 & 1.71 & 1.81 \\
\hline \multirow{2}{*}{$\begin{array}{l}\text { Confrontation }+ \text { Highlighting } \\
\text { resources }\end{array}$} & 6.65 & 7.87 & 6.65 & 7.93 \\
\hline & 2.19 & 1.64 & 2.34 & 1.65 \\
\hline
\end{tabular}

Note: Means are in bold and standard deviations in italics. 


\subsubsection{Perceived vitality}

An ANOVA found a main effect of condition $\left(F(5,785)=68.27 ; p<0.01 ; \eta p^{2}=.30\right)$ and a marginal main effect of gender $\left(F(1,785)=2.80 ; p=0.094 ; \eta p^{2}=.004\right)$ on perceived vitality. Planned contrasts revealed that, among the four interventions, perceived vitality was higher in the highlighting resources condition $(M=7.52, S D=1.76)$ than in the stating policy $(M=6.48, S D=2.15)$ and confrontation conditions $(M=6.87, S D=2.03)$ (both $p s<0.05)$ but not different than the hybrid condition $(M=7.27, S D=2.12)$. Perceived vitality was lowest in the no intervention condition ( $M$ $=3.75, S D=2.31$ ) compared to all other conditions (all $p \mathrm{~s}<0.001$ ). Again, perceived vitality was lower when there was no transgression $(M=5.15, S D=2.04)$ compared to any type of intervention post transgression (all $p s<0.001)$. Overall, women perceived marginally higher vitality $(M=6.30$, $S D=0.10)$ than men $(M=6.05, S D=0.10)$.

Again, there was a statistically significant interaction between gender and condition $(F(5$, $\left.785)=4.33 ; p<0.01 ; \eta p^{2}=.027\right)$. Women $(M=3.28, S E=0.26)$ perceived less vitality than men $(M=$ $4.18, S E=0.24)$ in the no intervention condition $(F(1,785)=6.52, p=.011)$ but perceived more vitality $(M=7.93, S E=0.26)$ than men $(M=6.65, S E=0.25)$ in the hybrid condition $(F(1,785)=$ 12.53, $p<0.001)$. See Table 1 for descriptive statistics and Figure 2 for interaction effects.

We found that all of the tested interventions were better than no intervention at all but among the interventions tested, highlighting resources led to the highest ratings of inclusion and vitality, especially among women.

\section{Figure 2. Perceived vitality in the relationship with the leader in Study 1}

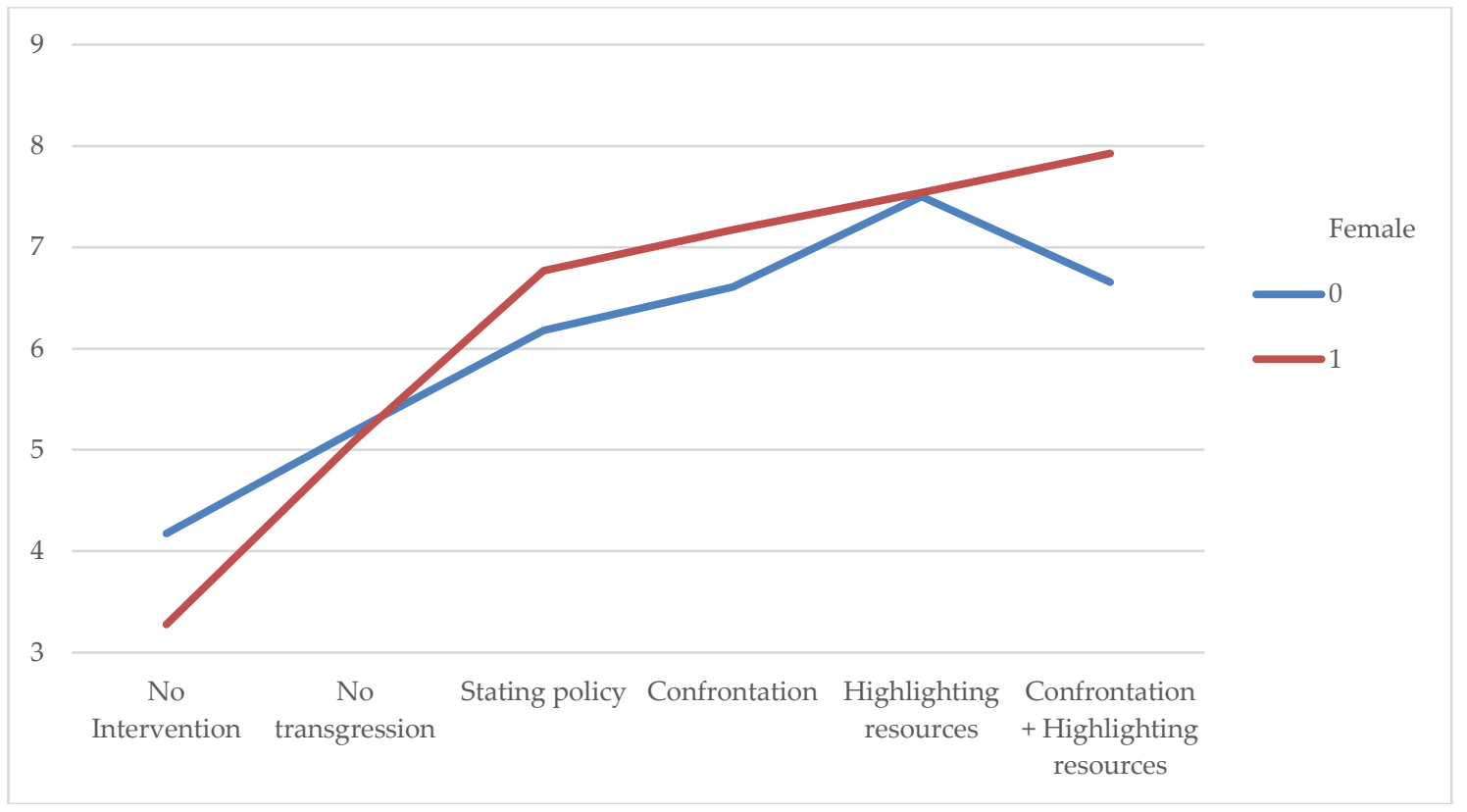

\subsubsection{Mediation via perceived inclusion}

To test if the relationship between intervention strategy and perceived vitality was mediated by perceived inclusion, we ran a mediation model (Model 4) using the PROCESS extension (Hayes, 2017). We entered the highlighting resources intervention conditions (including hybrid) combined versus all other conditions as the predictor variable, perceived vitality as the outcome variable and perceived inclusion as the mediator. Importantly, we found a statistically significant indirect effect of intervention on perceived vitality $[\mathrm{B}=1.71, S E=0.16,95 \%$ bootstrap CI [1.40, 2.01]). Thus, Study 1 results support that perceived vitality resulting from the intervention that highlights resources is mediated by perceived inclusion. See Figure 3 for path coefficients. 
Figure 3. Standardized path coefficients for mediation via perceived inclusion in Study 1

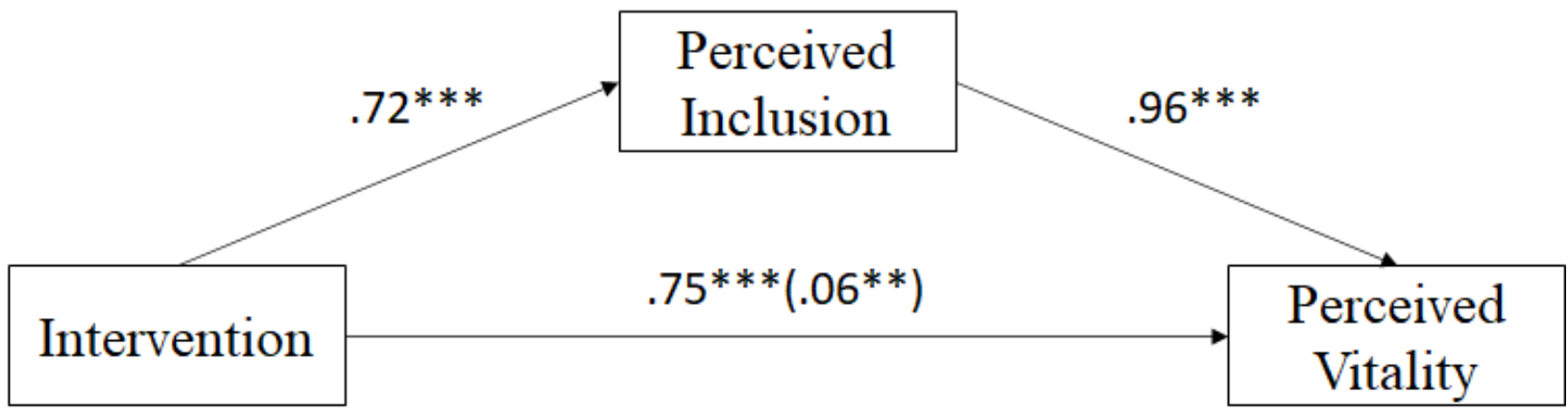

Note. ${ }^{*} p<.05 .{ }^{* *} p<.01 .{ }^{* * *} p<.001$

\section{Study 2: Differentiating types of resource highlighting}

In Study 1, we found that resource highlighting interventions (i.e., resource highlighting and hybrid) were perceived to create greater feelings of vitality by increasing feelings of inclusion. To extend results from this study, we further investigated the specific types of resources that can be highlighted by allies to maximize positive outcomes. Roberts and Cha (2015) suggest that an ally can highlight three broad types of cultural identity-based resources of a minority employee that range from observable, surface-level characteristics to non-observable yet significant, culturallyrelevant characteristics. Disentangling the type of resources that are highlighted based on their salience can enrich the extent to which a minority individual's impression is constructed, managed, and perceived by observers (Pfeffer et al., 2006).

To further offer practical guidelines for creating a parsimonious intervention, our goal for Study 2 was to explore the relative (dis)advantages of highlighting the three different types of resources: 1) symbolic capital, which is a resource that can be generated and leveraged through physical representation of diversity, 2) social capital, which is generated from the connections that marginalized group members can build with each other based on shared backgrounds and experiences, and 3) psychological and intellectual capital, which are affective and cognitive resources such as resilience and creativity that are generated through distinctive experiences (Roberts \& Cha, 2015). Specifically, we examined observers' perceptions of the impact of different types of resource highlighting-social capital, symbolic capital, and psychological and intellectual capital-on the wellbeing of marginalized employees in terms of inclusion and vitality.

\subsection{Method}

Eight hundred and three US-based workers (381 female, 421 male, and 1 other; Median age = 35) participated in an online panel for payment. The project received IRB exemption and participants provided informed consent. Sensitivity analyses indicate this sample size is sufficient to detect a small-to-medium effect size at $80 \%$ power (G*Power; Faul et al., 2007). After excluding data from one participant who chose 'other' for gender, the final sample size for this study was 802 participants. All participants read the same baseline scenario about a corporate meeting as in Study 1.

Again, participants were randomly assigned to one of the six between-subjects conditions (no transgression, no intervention, social capital highlighting, psychological and intellectual capital highlighting, symbolic capital highlighting, and all resource highlighting). The "no intervention" and "no transgression" conditions were the same as Study 1. See Appendix 2 for Study 2 stimuli. 
After reading the respective scenario, participants used a nine-point scale ranging from "not at all" to "very much" to indicate their inferences regarding Susan's feeling of vitality $(\alpha=0.98)$ and inclusion $(\alpha=0.97)$ in her relationship with the leader (same as Study 1), and their perceived sincerity of the leader's motives (e.g., "John responded the way he did in the meeting because he sincerely believes in confronting diversity and inclusion issues in the workplace"). Dasborough and Ashkanasy (2002) suggest that leadership behaviors are seen as 'true' (and not 'pseudo') if their motives behind those behaviors are seen as sincere as opposed to ulterior. Therefore, we designed this context-specific measure of perceived sincerity to explore if the interventions differ in terms of perceived sincerity and if these differences translate into differences in inclusion and vitality. Finally, participants answered demographic questions.

\subsection{Results}

\subsubsection{Perceived inclusion}

We found a main effect of condition $\left(F(5,790)=69.85, p<0.001, \eta^{2}=.31\right)$ on perceived inclusion. See Table 2 (below) for descriptive statistics and Figure 4 for the effects. Planned contrasts revealed that, among the four types of interventions, perceived inclusion was statistically significantly higher in the highlighting all resources condition $(M=7.46, S D=1.95)$ than the social capital $(M=6.69, S D=2.14)$ and symbolic capital condition $(M=6.74, S D=2.35$, both $p s<0.01)$ but not the psychological and intellectual capital condition $(\mathrm{M}=7.29, S D=1.71)$. Perceived inclusion was lowest in the no intervention condition $(M=3.59, S D=2.19)$ compared to all other conditions (all $p \mathrm{~s}<0.001)$. Perceived inclusion was lower when there was no transgression $(M=$ $5.15, S D=2.06$ ) as compared to any type of intervention post transgression (all $p \mathrm{~s}<0.001$ ).

\section{Figure 4. Perceived inclusion in the relationship with the leader in Study 2}

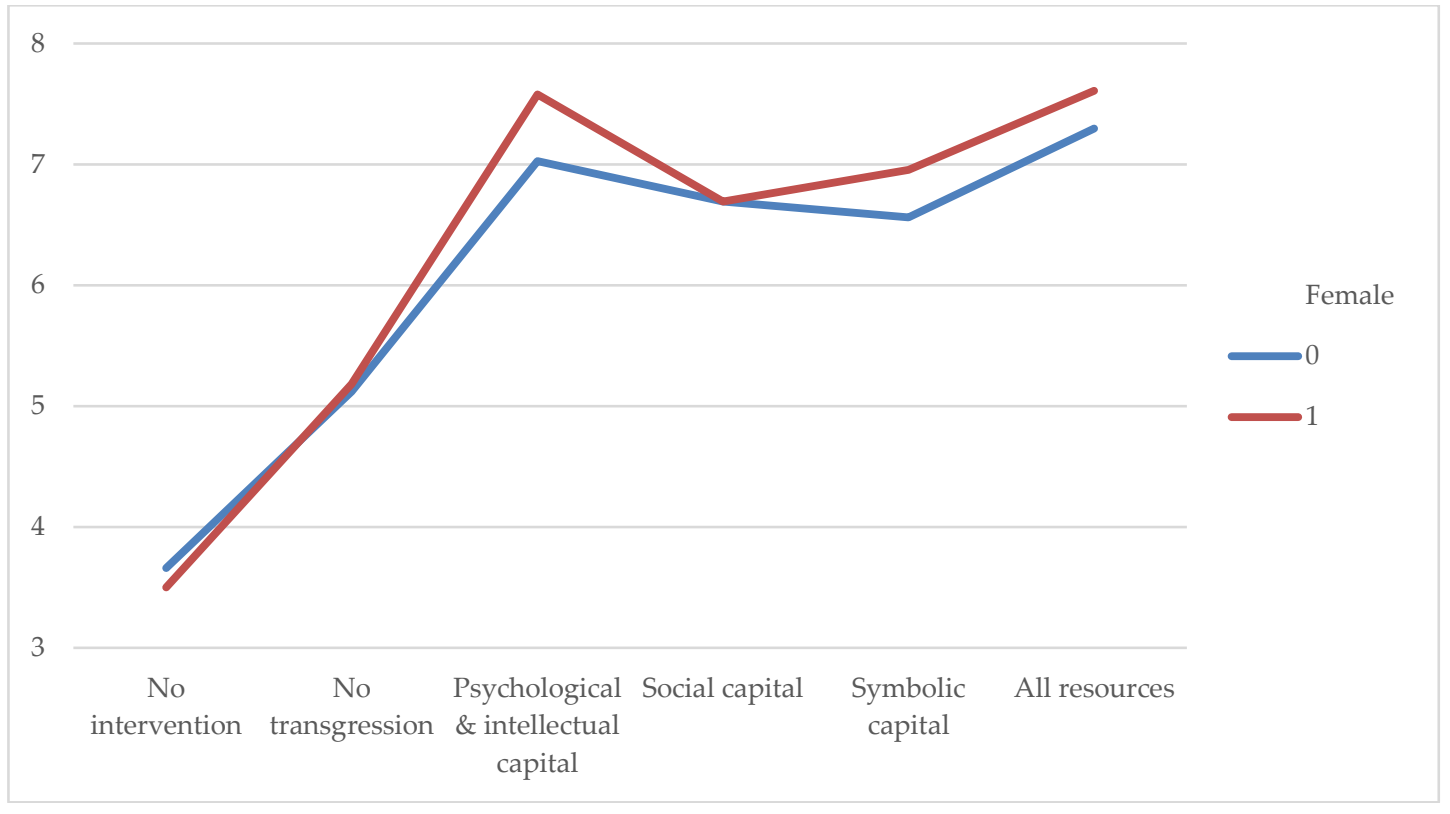


Table 2. Descriptive statistics for Study 2

\begin{tabular}{|c|c|c|c|c|c|c|}
\hline \multirow[t]{2}{*}{ Conditions } & \multicolumn{2}{|c|}{$\begin{array}{l}\text { Perceived } \\
\text { inclusion }\end{array}$} & \multicolumn{2}{|c|}{$\begin{array}{l}\text { Perceived } \\
\text { vitality }\end{array}$} & \multicolumn{2}{|c|}{$\begin{array}{l}\text { Perceived } \\
\text { sincerity }\end{array}$} \\
\hline & Male & Female & Male & Female & Male & Female \\
\hline \multirow[t]{2}{*}{ No intervention } & 3.66 & 3.50 & 3.60 & 3.50 & 3.27 & 2.73 \\
\hline & 2.06 & 2.35 & 1.99 & 2.50 & 2.17 & 2.58 \\
\hline \multirow[t]{2}{*}{ No transgression } & 5.12 & 5.18 & 4.92 & 5.04 & 4.29 & 4.69 \\
\hline & 2.01 & 2.13 & 2.01 & 2.15 & 2.28 & 2.42 \\
\hline \multirow{2}{*}{$\begin{array}{l}\text { Psychological \& } \\
\text { intellectual capital }\end{array}$} & 7.03 & 7.58 & 7.01 & 7.60 & 7.04 & 7.49 \\
\hline & 1.71 & 1.68 & 1.81 & 1.71 & 1.82 & 1.92 \\
\hline \multirow[t]{2}{*}{ Social capital } & 6.69 & 6.69 & 6.72 & 6.57 & 6.39 & 6.88 \\
\hline & 2.12 & 2.18 & 2.15 & 2.27 & 2.40 & 1.96 \\
\hline \multirow[t]{2}{*}{ Symbolic capital } & 6.56 & 6.96 & 6.50 & 6.90 & 6.12 & 6.55 \\
\hline & 2.26 & 2.44 & 2.26 & 2.37 & 2.32 & 2.47 \\
\hline \multirow[t]{2}{*}{ All resources } & 7.23 & 7.61 & 7.22 & 7.65 & 7.28 & 7.55 \\
\hline & 1.82 & 2.06 & 1.88 & 1.98 & 2.02 & 2.00 \\
\hline
\end{tabular}

Note: Means are in bold and standard deviations in italics

\subsubsection{Perceived vitality}

We found a main effect of condition $\left(F(5,790)=70.98, p<0.01 ; \eta p^{2}=.31\right)$ on perceived vitality. See figure 5. Planned contrasts revealed that, among the four types of interventions, perceived vitality was statistically significantly higher in the highlighting all resources condition $(M=7.44$, $S D=1.94)$ than in the social capital $(M=6.65, S D=2.20)$ and symbolic capital condition $(M=6.69$, $S D=2.31$, both $p s<0.01)$ but not the psychological and intellectual capital condition $(M=7.30, S D$ $=1.78)$. Perceived vitality was lowest in the no intervention condition $(M=3.56, S D=2.22)$ compared to all other conditions (all $p s<0.001$ ). Perceived vitality was lower when there was no transgression $(M=4.98, S D=2.07)$ as compared to any type of intervention post transgression (all $p \mathrm{~s}<0.001$ ). 
Figure 5. Perceived vitality in the relationship with the leader in Study 2

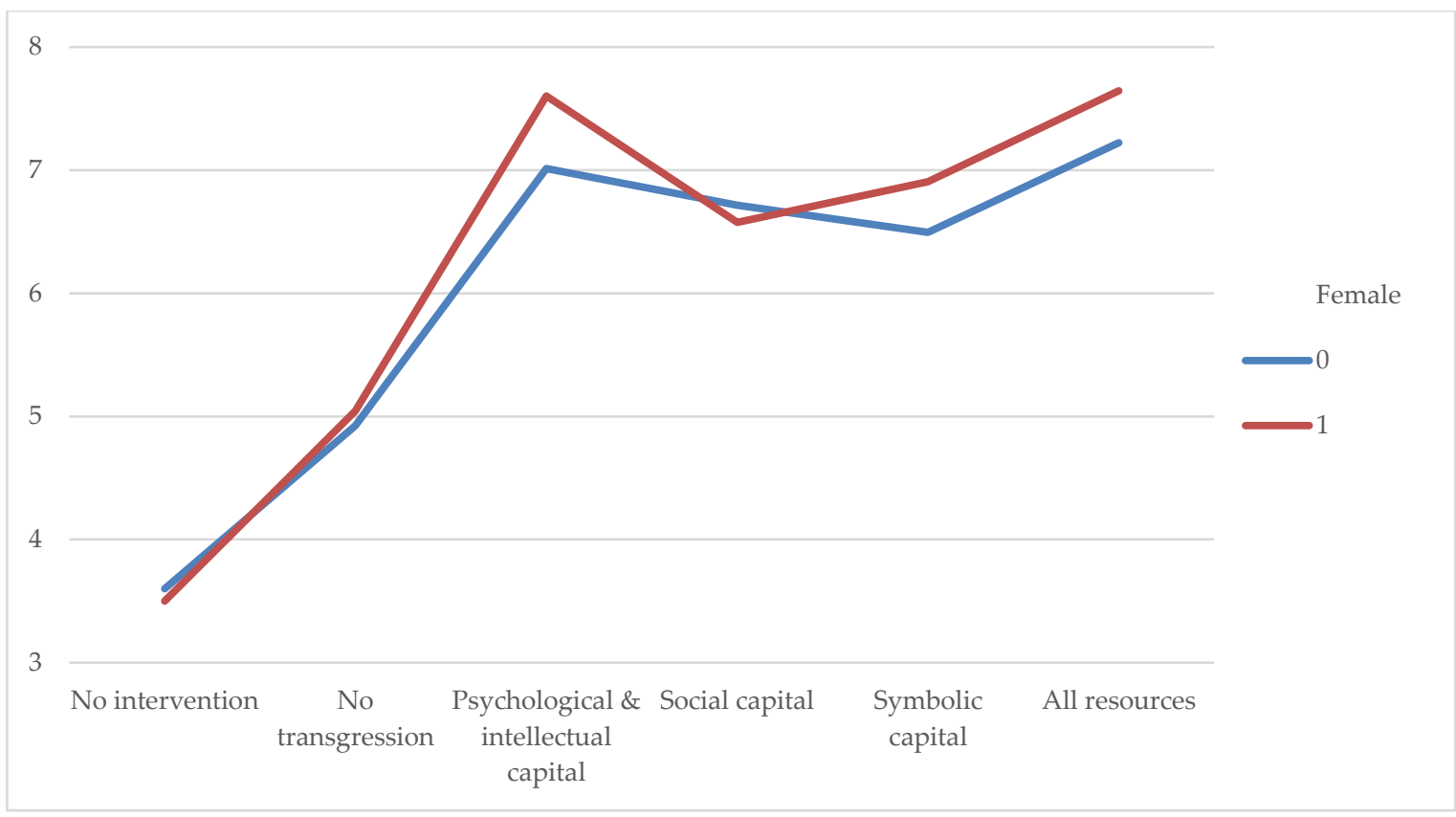

\subsubsection{Perceived sincerity of leader's motives}

We found a main effect of the condition $\left(F(5,790)=83.87, p<.001, \eta_{p}^{2}=.347\right)$ on perceived sincerity of the leader's motives, as shown in Figure 6. Planned contrasts revealed that, among the four types of interventions, perceived sincerity was statistically significantly higher in the highlighting all resources condition $(M=7.42, S D=2.01)$ than in the social capital $(M=6.63, S D$ $=2.21)$ and symbolic capital conditions $(M=6.32, S D=2.40$, both $p s<0.01)$ but not the psychological and intellectual capital condition $(M=7.26, S D=1.88)$. Perceived sincerity was lowest in the no intervention condition $(M=3.03, S D=2.37)$ compared to all other conditions (all $p s<0.001)$. Perceived sincerity was lower when there was no transgression $(M=4.48, S D=2.35)$ as compared to any type of intervention post transgression (all $p s<0.001$ ).

\section{Figure 6. Perceived sincerity of the motives of the leader in Study 2}

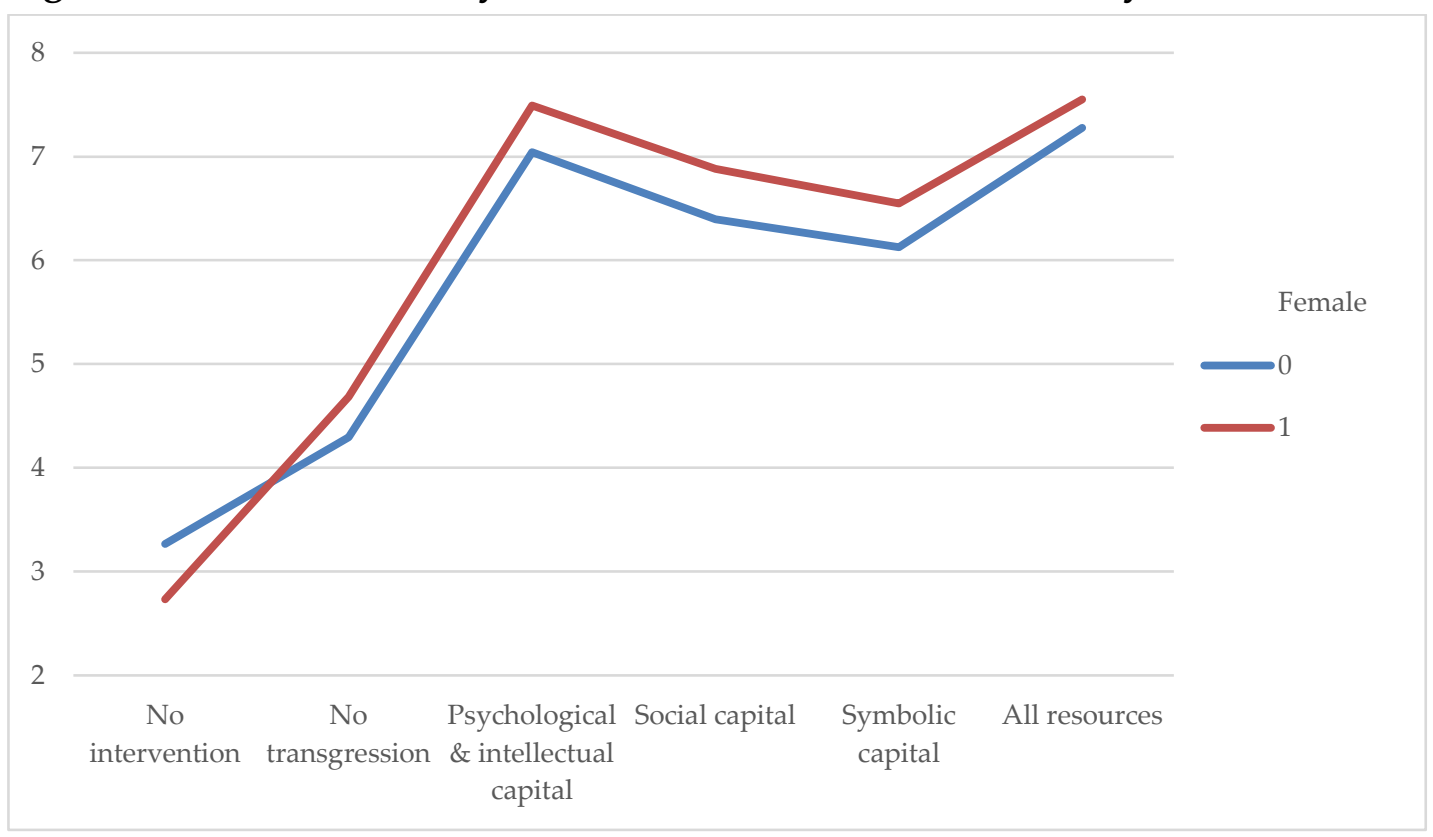




\subsubsection{Serial mediation via perceived sincerity and perceived inclusion}

To test whether the effect of an allyship intervention on perceived vitality was mediated by inferences related to perceived sincerity and perceived inclusion, we ran a serial mediation model (Model 6) using the SPSS PROCESS extension (Hayes, 2017). We entered highlighting all resources and highlighting only psychological and intellectual capital conditions combined versus all other conditions (including no intervention condition) as the predictor variable, perceived vitality as the outcome variable, and perceived sincerity and perceived inclusion as the two serial mediators. There was a statistically significant indirect effect of intervention on perceived vitality through perceived sincerity and perceived inclusion $(B=1.50, S E=0.12,95 \%$ bootstrap CI $[1.26,1.74])$. The direct effect was not statistically significant $(B=0.05, S E=0.04,95 \%$ CI $[-0.03,0.13])$, indicating a full mediation by perceived sincerity and perceived inclusion. See Figure 7 (below) for path coefficients. Thus, results from Study 2 show that highlighting psychological and intellectual capital as an intervention can lead to feelings of vitality if it is perceived as sincere because it increases feelings of inclusion.

Figure 7. Standardized path coefficients for serial mediation via perceived sincerity and perceived inclusion in Study 2

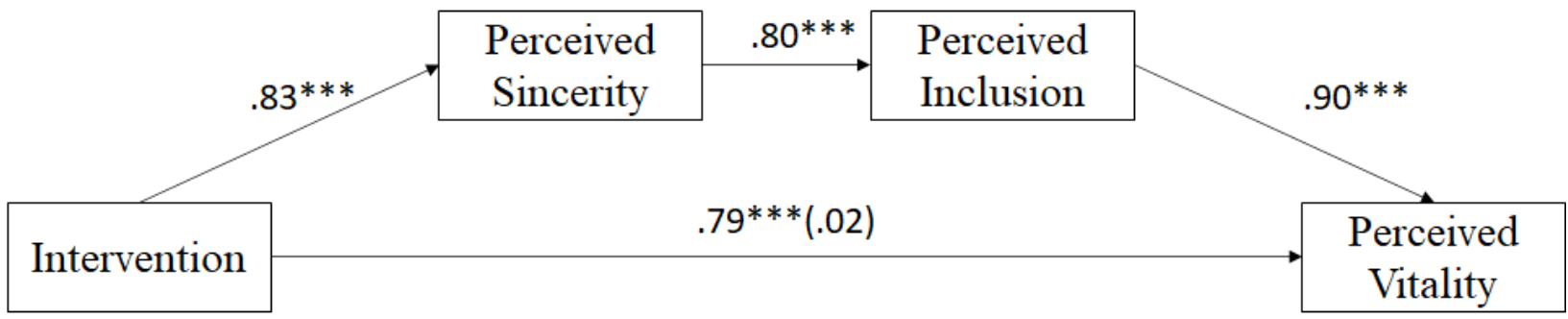

Note. ${ }^{*} p<.05 .{ }^{* *} p<.01 .{ }^{* * *} p<.001$

\subsubsection{Additional analyses}

Results from Study 2 showed that although highlighting all types of minority identity-related resources can be the best overall strategy for achieving positive outcomes, highlighting psychological and intellectual capital achieved equally positive results through more parsimonious means. To understand the distinction between highlighting psychological and intellectual capital as compared to highlighting social and symbolic capital, we ran a series of mediation analyses.

In our first model, we used highlighting psychological and intellectual capital (vs. social capital) as the independent variable, perceived vitality as the dependent variable and perceived sincerity as the mediator. There was only an indirect effect of type of intervention on perceived vitality through perceived sincerity $(\mathrm{B}=-0.44, S E=.18,95 \%$ CI [ $-0.81,-0.09])$. Similarly, we used highlighting psychological and intellectual capital (vs. social capital) as the independent variable, perceived inclusion as the dependent variable and perceived sincerity as the mediator. There was only an indirect effect of type of intervention on perceived inclusion through perceived sincerity $(\mathrm{B}=-0.42, \mathrm{SE}=.17,95 \% \mathrm{CI}[-0.78,-0.09])$.

In our second series of comparisons, we used highlighting psychological and intellectual capital (vs. symbolic capital) as the independent variable, perceived vitality as the dependent variable and perceived sincerity as the mediator. Again, there was only an indirect effect of type of intervention on perceived vitality through perceived sincerity $(B=-0.36, S E=.11,95 \% \mathrm{CI}$ [$0.57,-0.15]$ ). Similarly, when we used highlighting psychological and intellectual capital (vs. 
symbolic capital) as the independent variable, perceived inclusion as the dependent variable and perceived sincerity as the mediator, there was only an indirect effect of type of intervention on perceived inclusion through perceived sincerity $(B=-0.35, S E=.11,95 \% \mathrm{CI}[-0.58,-0.16])$.

Collectively, these findings show that perceived sincerity of the intervention accounts for the difference between highlighting psychological and intellectual resources which are internal to the marginalized individuals (and can be seen as desired ends in themselves) versus highlighting social and symbolic resources which could be seen as means to desirable ends and therefore, more extrinsically motivated, and less sincere.

\section{General discussion}

Across two studies, we investigated the extent to which various types of interventions used after a transgression were effective in eliciting feelings of inclusion and vitality, and found that the positive psychology-based strategy of resource highlighting was most effective. First, observers' responses indicated that all of the tested allyship interventions were perceived as effective in boosting the marginalized individual's feelings of inclusion and vitality post transgression, even compared to a situation where no transgression transpired. Thus, even though transgressive incidents against marginalized individuals can be injurious to the target individual, observers seem to believe that they provide an opportunity for allies to intervene and, when done effectively, allow allies to advocate for and enhance the cultural norms surrounding employees' identities. In the absence of a highly salient negative event such as a prejudiced transgression, it can be hard for an employee to make such inferences (Fiske, 1980), again reinforcing the opportunity that this intervention serves in setting organizational standards and norms.

Second, even though simply stating an official organizational policy regarding diversity and inclusion or confronting the exact transgression are valuable interventions compared to no intervention at all, highlighting the marginalized individual's resources was perceived to be the best strategy by observers. Highlighting resources can not only establish the sincerity and authenticity of the ally's motives but also carries the potential to contribute to the marginalized individual's feelings of inclusion and vitality.

Beyond simply suggesting a highlighting resources strategy, the results of our second study provide nuance regarding the type of resources that should be highlighted to be most effective. We found that highlighting marginalized individuals' psychological and intellectual capital was viewed by observers as an intervention that was just as effective in eliciting feelings of inclusion and vitality as highlighting all types of resources together (i.e., social and symbolic capital as well). Thus, targeting psychological and intellectual resources may serve as a more parsimonious yet equally effective strategy. Further, we demonstrated that highlighting psychological and intellectual capital is perceived to be successful because it enhances perceptions of the ally's sincerity. Although the positive psychology literature has demonstrated that leveraging personal social and symbolic capital in the workplace is important for personal wellbeing (e.g., Baker \& Dutton, 2007), our results suggest that leaders who highlight these resources in others may be seen as extrinsically motivated and not fully sincere in their commitment to confronting diversity and inclusion issues in the workplace. Therefore, leaders and allies need to be careful in terms of signaling the sincerity of their motives when enacting a resource highlighting intervention.

\subsection{Theoretical and practical implications}

At the interpersonal level, this research contributes to the existing scholarship on positive organizational interventions by showing that leaders can enact allyship by highlighting the resources of marginalized individuals, which signals to both marginalized group members and 
third-party observers how much the organization values the distinctive strengths of diverse individuals. This research provides allies and leaders with a quick, actionable, and nonconfrontational intervention that they can use to intervene following transgressions and to establish or change the organizational culture.

We do suggest that leaders receive training in highlighting resources to maximize its effectiveness and impact on wellbeing. In particular, careful development and application for highlighting resources is warranted. One concern is the potential misuse of highlighting a minority individual's identity in a way that perpetuates positive stereotypes, creating further negative impacts for these group members (e.g., Siy \& Cheryan, 2013, 2016). We caution against using generalities (e.g., mothers as nurturing) when identifying identity-relevant resources to highlight, and instead encourage a focus on employees' concrete strengths and abilities (e.g., ability to relate to other mothers who juggle responsibilities in the organization) that accrue from specific experiences (e.g., juggling multiple responsibilities). Ideally, leaders using this intervention should learn about the specific identity-relevant strengths and achievements (i.e., individuating information such as head of women's leadership network) of marginalized employees so that this information is readily accessible when there is a need (i.e., to counter a microaggression) to highlight them.

\subsection{Limitations and future research}

A limitation of this work is the reliance on scenario-based experiments to evaluate perceptions of vitality and inclusion. Future research should attempt to replicate these findings using a field study or realistic experiment with confederates. However, because this research was aimed at developing and testing a novel intervention, we believe that our design was able to minimize potential biases that affect realistic paradigms (e.g., demand characteristics, experimenter effects; Bracht \& Glass, 1968). The focal individual in this experiment was a female, limiting the generalizability to other marginalized groups. The results could be generalized if the studies were repeated with the identity of the focal individual varied (e.g., to a racial minority). Finally, a leader's acknowledgment of strengths via individuating information serves to signal that one is valued and possesses promotable strengths. As such, it would be useful for future research to examine whether resource highlighting produces positive effects in the absence of a transgression. If implementing this strategy serves to promote a positive and inclusive environment more generally, leaders could potentially use this framework as a preventative strategy against prejudiced events.

\section{Conclusion}

Leaders and individuals from dominant groups are increasingly expressing discomfort with prejudice and discrimination, but in the face of a transgression, are unsure how to intervene in the moment due to concerns about how they would be perceived (Warren et al., 2022). Intentions are not enough, though, and leaders need concrete interventions that work and preferably, those that are perceived positively by others. The current studies offer such concrete guidance to leaders who desire to be active allies. We compared and tested interventions that leader-allies can employ immediately after they witness prejudiced behavior that will incur positive effects rather than solely negative. We found that although all of the tested interventions were better than no intervention, PPIs involving highlighting the distinctive identity-based strengths of minority individuals were viewed as the most effective. Our hope is that this work will inspire other scholars to consider developing theoretically grounded PPIs and practitioners to use such 
interventions that have the capacity to generate wellbeing for targets of discrimination and create a positive organizational culture.

\section{Conflict of interest statement}

The authors declared no potential conflicts of interest with respect to the research, authorship, and/or publication of this article.

\section{Funding Statement}

This research was supported by two small grants from Western Washington University.

\section{Data Availability Statement}

Data is available upon request via the corresponding author.

\section{Authors}

Meg A. Warren

Western Washington University

Meg.Warren@wwu.edu

Tejvir Sekhon

Western Washington University

Rachael J. Waldrop

Western Washington University

\section{Authors' Contributions}

M.W. and T.S. are equal first authors.

\section{Acknowledgments}

Special thanks to Natalie Sappleton for her invaluable feedback on previous drafts of this manuscript.

\section{Publishing Timeline}

Received 30 May 2021

Revised version received 29 January 2022

Accepted 30 January 2022

Published 31 January 2022

\section{References}

Ashburn-Nardo, L., Morris, K. A., \& Goodwin, S. A. (2008). The Confronting Prejudiced Responses (CPR) Model: Applying CPR in organizations. Academy of Management Learning \& Education, 7(3), 332-342. https://doi.org/10.5465/AMLE.2008.34251671

Baba, Y., \& Hebert, C. (2004). The effects of participation in a cultural awareness program on jail inmates. Journal of Ethnic \& Cultural Diversity in Social Work: Innovation in Theory, Research \& Practice, 13(3), 91113. https://doi.org/10.1300/J051v13n03_05

Baker, W. \& Dutton, J. (2007). Enabling positive social capital in organizations. In J. Dutton and B. Ragins (Eds.). Exploring positive relationships at work: Building a theoretical and research foundation (pp. 325-346). New Jersey: Lawrence Erlbaum, Inc.

Bakker, A. B., Petrou, P., Op den Kamp, E. M., \& Tims, M. (2020). Proactive vitality management, work engagement, and creativity: The role of goal orientation. Applied Psychology, 69(2), 351-378. https://doi.org/10.1111/apps.12173. 
Bracht, G. H., \& Glass, G. V. (1968). The External Validity of Experiments. American Educational Research Journal, 5(4), 437-474. https://doi.org/10.3102/00028312005004437

Brooks, A. K., \& Edwards, K. (2009). Allies in the workplace: Including LGBT in HRD. Advances in Developing Human Resources, 11(1), 136-149.

Buengeler, C., Leroy, H., \& De Stobbeleir, K. (2018). How leaders shape the impact of HR's diversity practices on employee inclusion. Human Resource Management Review, 28(3), 289-303. https://doi.org/10.1016/j.hrmr.2018.02.005

Carmeli, A. (2009). Positive work relationships, vitality, and job performance. In C. Härtel, N. Ashkanasy, \& W. Zerbe (Eds.), Research on emotion in organizations: Vol. 5. Emotions in groups, organizations and cultures (pp. 45-71). Emerald Group Publishing Limited.

Cha, S. E., \& Roberts, L. M. (2019). Leveraging minority identities at work: An individual-level framework of the identity mobilization process. Organization Science, 30(4), 735-760.

Cha, S. E., Hewlin, P. F., Roberts, L. M., Buckman, B. R., Leroy, H., Steckler, E. L., ... \& Cooper, D. (2019). Being your true self at work: Integrating the fragmented research on authenticity in organizations. Academy of Management Annals, 13(2), 633-671.

Chaney, K. E., \& Sanchez, D. T. (2018). The endurance of interpersonal confrontations as a prejudice reduction strategy. Personality and Social Psychology Bulletin, 44(3), 418-0429. https://doi.org/10.1177/0146167217741344

Crary, M. (2017). Working from dominant identity positions: Reflections from "diversity-aware" white people about their cross-race work relationships. The Journal of Applied Behavioral Science, 53(2), 290316. https://doi.org/10.1177/0021886317702607

Creary, S. J., Caza, B. B., \& Roberts, L. M. (2015). Out of the box? How managing a subordinate's multiple identities affects the quality of a manager-subordinate relationship. Academy of Management Review, 40(4), 538-562.

Czopp, A. M., \& Monteith, M. J. (2003). Confronting prejudice (literally): Reactions to confrontations of racial and gender bias. Personality and Social Psychology Bulletin, 29(4), 532-544.

Czopp, A. M., Monteith, M. J., \& Mark, A. Y. (2006). Standing up for a change: Reducing bias through interpersonal confrontation. Journal of Personality and Social Psychology, 90(5), 784-803. https://doi.org/10.1037/0022-3514.90.5.784

Dasborough, M. T., \& Ashkanasy, N. M. (2002). Emotion and attribution of intentionality in leadermember relationships. The Leadership Quarterly, 13(5), 615-634.

Den Hartog, D. N., Boon, C., Verburg, R. M., \& Croon, M. A. (2013). HRM, communication, satisfaction, and perceived performance: A cross-level test. Journal of Management, 39(6), 1637-1665. https://doi.org/10.1177/0149206312440118

DeTurk, S. (2011). Allies in action: The communicative experiences of people who challenge social injustice on behalf of others. Communication Quarterly, 59(5), 569-590.

Ellis, A. M., Bauer, T. N., Erdogan, B., \& Truxillo, D. M. (2019). Daily perceptions of relationship quality with leaders: Implications for follower well-being. Work \& Stress, 33(2), 119-136.

Erskine, S. E., \& Bilimoria, D. (2019). White allyship of Afro-Diasporic women in the workplace: A transformative strategy for organizational change. Journal of Leadership \& Organizational Studies, 26(3), 319-338. https://doi.org/10.1177/1548051819848993

Faul, F., Erdfelder, E., Lang, A.-G., \& Buchner, A. (2007). G*Power 3: A flexible statistical power analysis program for the social, behavioral, and biomedical sciences. Behavior Research Methods, 39(2), 175-191. https://doi.org/10.3758/ BF03193146

Fiske, S. T. (1980), Attention and weight in person perception: The impact of negative and extreme behavior. Journal of Personality and Social Psychology, 38 (6), 889-906.

Fisk, G. M., \& Friesen, J. P. (2012). Perceptions of leader emotion regulation and LMX as predictors of followers' job satisfaction and organizational citizenship behaviors. The Leadership Quarterly, 23(1), 112.

Foster, M. D. (2009). Perceiving pervasive discrimination over time: Implications for coping. Psychology of Women Quarterly, 33(2), 172-182. 
Gulker, J. E., Mark, A. Y \& Monteith, M. J. (2013). Confronting prejudice: The who, what, and why of confrontation effectiveness, Social Influence, 8(4), 280-293.

Hayes, A. F. (2017). Introduction to mediation, moderation, and conditional process analysis. New York, NY: Guilford Publications, Inc

Hood, J. N., Muller, H. J., \& Seitz, P. (2001). Attitudes of Hispanics and Anglos surrounding a workforce diversity intervention. Hispanic Journal of Behavioral Sciences, 23(4), 444-458. https://doi.org/10.1177/0739986301234006

Jansen, W. S., Otten, S., van der Zee, K. I., \& Jans, L. (2014). Inclusion: Conceptualization and measurement. European Journal of Social Psychology, 44(4), 370-385. doi:10.1002/ejsp.2011

Jeglic, E. L., Miranda, R., \& Polanco-Roman, L. (2016). Positive psychology in the context of race and ethnicity. In E. C. Chang, C. A. Downey, J. K. Hirsch, \& N. J. Lin (Eds.), Cultural, racial, and ethnic psychology book series. Positive psychology in racial and ethnic groups: Theory, research, and practice (p. 1333). American Psychological Association. https://doi.org/10.1037/14799-002

Kark, R., \& Carmeli, A. (2009). Alive and creating: The mediating role of vitality and aliveness in the relationship between psychological safety and creative work involvement. Journal of Organizational Behavior, 30(6), 785-804.

Kidder, D. L., Lankau, M. J., Chrobot-Mason, D., Mollica, K. A., \& Friedman, R. A. (2004). Backlash toward diversity initiatives: Examining the impact of diversity program justification, personal and group outcomes. International Journal of Conflict Management, 15(1), 77-102. doi:10.1108/eb022908

Lambert, L., Warren, M. A., Brulé, G., O’Brien, C., Murray, S., Mulay-Shah, A., Passmore, H-A., Zelenski, J. M., Asfour, M., \& Alsubaiei, S. (2020). Perspectives: Using positive psychology and the United Nations' Sustainable Development Goals to build a better world. Middle East Journal of Positive Psychology, 6, 1-28.

Major, B., \& Schmader, T. (1998). Coping with stigma through psychological disengagement. In J. K. Swim \& C. Stangor (Eds.), Prejudice: The target's perspective. (pp. 219-241). San Diego, CA: Academic Press.

Mallett, R. K., \& Monteith, M. J. (Eds.). (2019). Confronting prejudice and discrimination: The science of changing minds and behaviors. Academic Press.

Martinez, L. R., Hebl, M. R., Smith, N. A., \& Sabat, I. E. (2017). Standing up and speaking out against prejudice toward gay men in the workplace. Journal of Vocational Behavior, 103(Part A), 71-85. https://doi.org/10.1016/j.jvb.2017.08.001

Meyers, M. C., van Woerkom, M., \& Bakker, A. B. (2013). The added value of the positive: A literature review of positive psychology interventions in organizations. European Journal of Work $\mathcal{E}$ Organizational Psychology, 22(5), 618-632. https://doi.org/10.1080/1359432X.2012.694689

Pascoe, E. A., \& Richman, L. S. (2009). Perceived discrimination and health: A meta-analytic review. Psychological Bulletin, 135(4), 531-554. https://doi.org/10.1037/a0016059

Pfeffer, J., Fong, C. T., Cialdini, R. B., \& Portnoy, R. R. (2006). Overcoming the self-promotion dilemma: Interpersonal attraction and extra help as a consequence of who sings one's praises. Personality and Social Psychology Bulletin, 32(10), 1362-1374.

Purcell, J. and Hutchinson, S. (2007), Front-line managers as agents in the HRM-performance causal chain: theory, analysis and evidence. Human Resource Management Journal, 17, 3-20. doi: https://doi.org/10.1111/j.1748-8583.2007.00022.x

Rao, M. A., \& Donaldson, S. I. (2015). Expanding opportunities for diversity in positive psychology: An examination of gender, race, and ethnicity. Canadian Psychology, 56(3), 271-282. https://doi.org/10.1037/cap0000036

Rasinski, H. M., Geers, A. L., \& Czopp, A. M. (2013). 'I guess what he said wasn't that bad': Dissonance in nonconfronting targets of prejudice. Personality and Social Psychology Bulletin, 39(7), 856-869. https://doi.org/10.1177/0146167213484769

Roberts, L. M., \& Cha, S. (2015). Sources of strength: mobilizing minority racial, ethnic, and cultural identities as resources. In Positive Organizing in a Global Society (pp. 57-62). Routledge. 
Ryan, R. M., \& Frederick, C. (1997). On Energy, Personality, and Health: Subjective Vitality as a Dynamic Reflection of Well-Being. Journal of Personality, 65(3), 529-565. https://doi.org.ezproxy.library.wwu.edu/10.1111/j.1467-6494.1997.tb00326.x

Shore, L. M., Randel, A. E., Chung, B. G., Dean, M. A., Ehrhart, K. H., \& Singh, G. (2011). Inclusion and diversity in work groups: A review and model for future research. Journal of Management, 37, 12621289.

Siy, J. O., \& Cheryan, S. (2013). When compliments fail to flatter: American individualism and responses to positive stereotypes. Journal of Personality and Social Psychology, 104(1), 87-102. https://doi.org/10.1037/a0030183

Siy, J. O., \& Cheryan, S. (2016). Prejudice masquerading as praise: The negative echo of positive stereotypes. Personality and Social Psychology Bulletin, 42(7), 941-954.

Sue, D. W. (2017). Microaggressions and "evidence" empirical or experiential reality?. Perspectives on Psychological Science, 12(1), 170-172.

Triana, M. del C., Jayasinghe, M., \& Pieper, J. R. (2015). Perceived workplace racial discrimination and its correlates: A meta-analysis. Journal of Organizational Behavior, 36(4), 491-513. https://doi.org/10.1002/job.1988

Vaughan, M. D., Miles, J., Parent, M. C., Lee, H. S., Tilghman, J. D., \& Prokhorets, S. (2014). A content analysis of LGBT-themed positive psychology articles. Psychology of Sexual Orientation and Gender Diversity, 1(4), 313.

Warren, M.A., \& Bordoloi, S.D. (2020). When COVID-19 exacerbates inequities: The path forward for generating wellbeing. International Journal of Wellbeing, 10(3), 1-6. doi:10.5502/ijw.v10i3.1357

Warren, M.A., \& Bordoloi, S.D. (2021). Going beyond good colleagues: Men's and women's perspectives on allyship behaviors toward women faculty in male-dominated disciplines in academia. Journal of Diversity in Higher Education. https://doi.org/10.1037/dhe0000369

Warren, M. A., Bordoloi, S. D., \& Warren, M. T. (2021). Good for the goose AND good for the gander: Examining positive psychological benefits of male allyship for men and women. Psychology of Men and Masculinities. https://doi.org/10.1037/men0000355

Warren, M. A., Donaldson, S. I., Lee, J. Y., \& Donaldson, S. I. (2019). Reinvigorating research on gender in the workplace using a positive work and organizations perspective. International Journal of Management Reviews, 21(4), 498-518. https://doi.org/10.1111/ijmr.12206

Warren, M. A. \& Schwam, A. (2021). So you think you are an ally? Effects of (in)congruence between men's and women's perceptions of allyship on women's inclusion and vitality. [Preprint] PsyArXiv. https://doi.org/10.31234/osf.io/ajgfm

Warren, M. A., Sekhon, T., Winkelman, K. M., \& Waldrop, R. J. (2021). 'Should I 'check my emotions at the door?' or express how I feel? Role of emotional regulation versus expression by authority figures speaking out against prejudice in the workplace. [Preprint] PsyArXiv. https://doi.org/10.31234/osf.io/jzxmy

Warren, M.A., Winkelman, K. M., \& Waldrop, R. J. (2022). Emotion management by organizational leaders who confront prejudice: Exploring emotion and social regulation of allies. [Preprint] PsyArXiv. https://doi.org/10.31234/osf.io/crnzp

Washington, J., \& Evans, N. J. (1991). Becoming an ally. In N. J. Evans, \& V. A. Wall (Eds.), Beyond tolerance: Gays, lesbians and bisexuals on campus (pp. 195-204). Alexandria, VA: American Association for Counseling and Development.

Waters, L. E., Cameron, K., Nelson-Coffey, S.K., Crone, D. L., Kern, M.L., Lomas, T., Oades, L., Owens, R.L., Pawelski, J. O., Rashid, T., Warren, M. A., White, M., Williams, P. (2021). Collective wellbeing and post traumatic growth during COVID-19: How positive psychology can help families, schools, workplaces, and marginalized communities. Journal of Positive Psychology. https://doi.org/10.1080/17439760.2021.1940251

Yakushko, O., \& Blodgett, E. (2021). Negative reflections about positive psychology: On constraining the field to a focus on happiness and personal achievement. Journal of Humanistic Psychology, 61(1), 104131. 


\section{Appendix}

\section{Appendix A: Study 1 Stimuli}

No transgression condition:

After everybody congratulated Susan, John (the VP) responded- "Well, let's move on to the agenda for the day." Then, he moved on to the agenda for the meeting."

\section{No intervention condition:}

As everybody started congratulating Susan, David blurted, "Again? Congrats I guess, but that's surely going to be the end of your career." On hearing this, John (the VP) responded- "Well, let's move on to the agenda for the day." Then, he moved on to the agenda for the meeting.

\section{Stating policy condition:}

As everybody started congratulating Susan, David blurted, "Again? Congrats I guess, but that's surely going to be the end of your career." On hearing this, John (the VP) responded- "Well, at FitWell Shoes, we are committed to equal opportunity and do not discriminate based on pregnancy." Then, he moved on to the agenda for the meeting.

\section{Confrontation condition:}

As everybody started congratulating Susan, David blurted, "Again? Congrats I guess, but that's surely going to be the end of your career." On hearing this, John (the VP) responded, "Well, I have four kids, and no one has said any such thing to me. Your comment is a little sexist, don't you think?"

\section{Resource highlighting condition:}

As everybody started congratulating Susan, David blurted, "Again? Congrats I guess, but that's surely going to be the end of your career." On hearing this, John (the VP) responded, "Well, I think this is great for Susan - personally and professionally! Her experiences as a parent will make her a better leader and prepare her for juggling multiple responsibilities, being more patient with customers, and building relationships with our diverse customer base. She will also be a great role model for our current and prospective female employees helping us build more diverse and creative teams.

\section{Hybrid (Confrontation + resource highlighting) condition:}

As everybody started congratulating Susan, David blurted, "Again? Congrats I guess, but that's surely going to be the end of your career." On hearing this, John (the VP) responded, "Well, I think this is great for Susan - personally and professionally! Her experiences as a parent will make her a better leader and prepare her for juggling multiple responsibilities, being more patient with customers, and building relationships with our diverse customer base. She will also be a great role model for our current and prospective female employees helping us build more diverse and creative teams. Moreover, I have four kids, and no one has said any such thing to me. Your comment is a little sexist, don't you think?" 


\section{Appendix B: Study 2 Stimuli}

\section{Highlighting social capital condition:}

As everybody started congratulating Susan, David blurted, "Again? Congrats I guess, but that's surely going to be the end of your career." On hearing this, John (the VP) responded, "Well, I think this is great for Susan - personally and professionally as well as great for the organization! As an executive who is also a mother of two kids, she will be able to connect with and build relationships with other female employees experiencing motherhood.

\section{Highlighting psychological and intellectual capital condition:}

As everybody started congratulating Susan, David blurted, "Again? Congrats I guess, but that's surely going to be the end of your career." On hearing this, John (the VP) responded, "Well, I think this is great for Susan - personally and professionally as well as great for the organization! Being an executive who is also a mother of two kids will broaden her perspective and juggling multiple responsibilities will make her more resilient.

Highlighting symbolic capital condition:

As everybody started congratulating Susan, David blurted, "Again? Congrats I guess, but that's surely going to be the end of your career." On hearing this, John (the VP) responded, "Well, I think this is great for Susan - personally and professionally as well as great for the organization! As an executive who is also a mother of two kids, she will be a great role model for our current and prospective female employees.

\section{Highlighting all resources condition:}

As everybody started congratulating Susan, David blurted, "Again? Congrats I guess, but that's surely going to be the end of your career." On hearing this, John (the VP) responded, "Well, I think this is great for Susan - personally and professionally as well as great for the organization! Being an executive who is also a mother of two kids will broaden her perspective and juggling multiple responsibilities will make her more resilient. She will also be a great role model for our current and prospective female employees and will be able to connect with and build relationships with other female employees experiencing motherhood. 